\title{
MEDIA KNOWLEDGE IN DIGITAL TRANSFORMATION: CONCEPTS AND MEANINGS OF FICTIONAL DIGITAL STORYTELLING
}

\author{
Victoria Tze Lin Kuan \\ Tunku Abdul Rahman University College, Malaysia
}

\begin{abstract}
Historically, art and beauty are classified and tagged. The classes and tags are the major limitations of human's weaknesses in confronting, representing and communicating the subjects and entity of reality in the physical world. The challenge of this paper is to investigate these intellectual search dimensions of human quest in managing interpretations specifically in the arts and science of perfecting storytelling knowledge in this digital era. To empower our role as audience of today's shared content, we need to question the study for the state of digital transformation processes that digital "storytellers" are facing in this intellectual search. This paper aims to investigate and tap into that specific knowledge area, and help identifies and analyze steps of critical conditioning of the digital landscape transformation processes, as we play the role of blended content creators and engage at the same time as media content audience during stories creations and expressions sharing processes. This paper discusses the models for development of fictional storytelling concepts, examines the real-world fictional stories representation mapping steps, appropriating digitalstorytelling tasks, identifying problems in knowledge representations (creator's input) and interpretations (audience perception) and reflecting on digital world manifestation modes as possible solutions. Investigations present contributing factors for content stories representations that will infer new insights of knowledge during media content creations and development processes. Conclusion will be drawn from theoretical and experiential study insights discussing the changing needs of recognizing media, not only as "windows" for stories expressions to audience. The focus will elaborate subjective issues designers and marketing content developers are facing, aiming to draw findings to re-position content "design thinking" as "elements" for media change and value creations in digital economies context.
\end{abstract}

Keywords: Digital transformation, digital storytelling, value creations, digital economies

\section{INTRODUCTION}

\section{Background}

In Part II of the book "Homo Deus" by Harari (2017, pp.180-181), he described how human have become convinced that they not only control the world, but at the same time gave the world its own meaning. He also questioned about the perspectives that were based on humanity and human's power of storytelling, the skills that human for generations have self-taught to "give meaning to the world" in order to survive. This research paper is to investigate the humanistic aspects of human's interpretation of the world in relations to representation and expression of "self" in that world; how relevant or capable they are to represent intellectual knowledge, whether in objective forms and or with added dimensions of fictional settings in digital context. It also aims to satisfy human quest and capability in telling stories by actualizing human experience in this physical reality. The knowledge gap that this investigation perspective is, to question how human see the world, and the search of the knowledge in seeing and in "mirroring" themselves in that attached stories. Technically, it is the representational dimensions that author aimed to explore, by discussing methods in appropriating real-world storytelling knowledge and tasks. The results from this research aim to present findings that will help designers, media 
professionals and content audience to recognize and reflect on the importance of digital storytelling tasks that can bring values to digital economies. The overall main focus is in exploring, discussing and redefining meanings of storytelling and representations, by proposing methods and models for organization of communication information, emphasizing more on practical methods that help classifying various storytelling goals when constructing represented content for digital and social media contexts.

\section{Research concepts}

Historically, art and beauty are classified and tagged, and many researchers have explored art as a form of aesthetics to define and make sense of our world (Whitehead, 1967, pp.241-243). It is a basic conceptual challenge to accept the fact that these classifications and tags are, however, a major limitation of human weaknesses in confronting, representing and communicating the subjects and entities of reality in the physical world. In his definitive writing for “Art in its relations", Croce (1976, pp. 561-562) mentioned that, “...the category of art, like every other category, mutually presupposes and is presupposed by all the rest: it is conditioned by them all and conditions them all". The challenge of this paper is to investigate these intellectual search dimensions of human quest in managing interpretations, specifically in the arts and science of perfecting storytelling knowledge in this digital era (Croce, 1976, pp. 559-560).

Storytelling, is a form of human communication that human attempts to make sense of the world (Harari, 2017, pp. 180-181). Also, storytelling processes are in fact a command of knowledge processes that require human leveraging the techniques of communication in conceptual approaches. As defined by Croce, human communication is "the fixation of the intuition-expression upon an object metaphorically called material or physical" and he stressed that "in reality, [...] we are concerned not with material or physical things but with a mental process" (1976, p. 566).

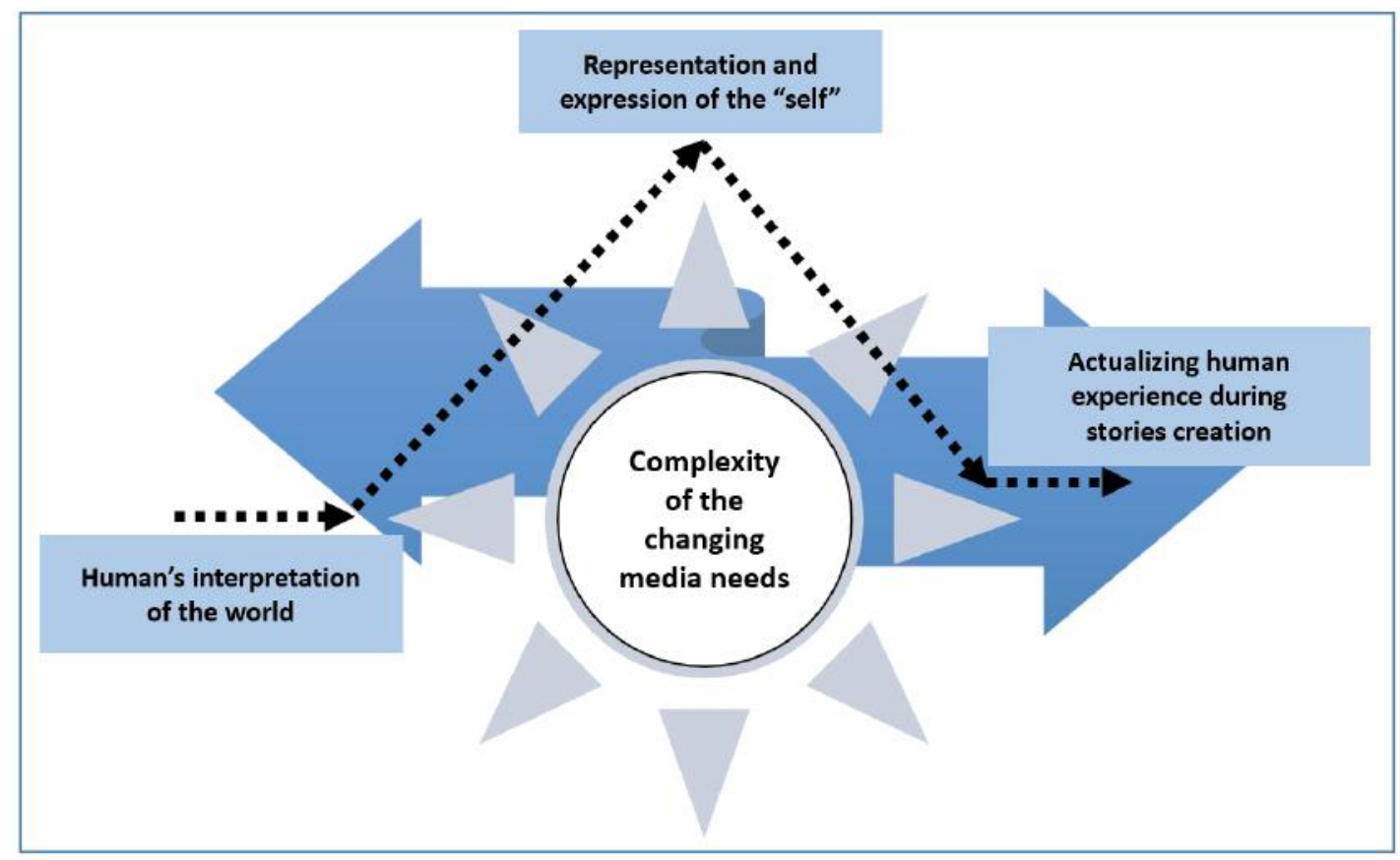

Figure 1 Preliminary conceptual model ${ }^{1}$ showing human storytelling experience drawing on a complexity of media needs to perfect storytelling skills in reality world.

${ }^{1}$ Figure 1 A hypothetical model created with reference to Freytag's dramatic story structure Pyramid model; Case example, human actualizing storytelling experience was evidential as demonstrated in a musical performance by an artist, Alexandro Querevalu, available at https://www.youtube.com/watch? $v=H X a g l T F J L M c \& f e a t u r e=$ share 


\section{RESEARCH OBJECTIVES}

Seeing the needs to answer to the abovementioned intellectual search, this paper proposed that it is absolutely important and essential for us in this historical context, and from this twenty first century perspective, to question on how we can empower our roles as audiences of today's shared content in digital context. By answering to this research goal, we need to question the study for the state of digital transformation processes that digital "storytellers" are facing in the intellectual search in real world context. The core areas of exploration and objectives of this paper encompassed to provide theoretical and elaborative discussion on: (1) Knowledge representation - to discuss various models for the development of fictional storytelling concepts; (2) Engagement processes - to examine the objective-fictional stories representation and mapping issues, and (3) New conceptualized definitions and meanings: appropriating digital-storytelling tasks and identifying problems in knowledge representations (creator's input) and interpretations (audience perception) modes as possible solutions in future state of digital landscape.

\section{Investigation Perspectives}

To examine the stated objectives, this paper will provide a discussion on the methods for investigations that will help to identify and examine relevant contributing factors for content stories representations. Consequentially, it explores the motivations of the proposed models that will help researchers explore the dimensions in powerful mind-changing and mind-shaping storytelling engagement with our audience in this new digital era. The studies from these intellectual activities will be projected to help researcher better illustrates their work in digital storytelling.

The abovementioned goals and perspectives of the study is to identify needs and knowledge gaps to enhance human understanding in perfecting our storytelling processes especially in terms of practical digital world context. Discussions of this study in this aspect will therefore draw inference on presenting useful insights at particular knowledge cycle processes during the media content creations and development processes in the storytelling activities.

\section{Research Methods}

This research study will focus on investigation on specific aspects of human's interpretation of the world in relations to the content knowledge representation and the expressions of the creator-"self" in that world; Therefore, it seems very much that we are potentially positioned to be on the intellectual search to make sense of the questioning roles of our media audience engaging in the 'interplays' effected with media complexities evidenced in its changing media needs from today's audience and those digitally social-elite citizens. In other words, it is the shaping of the media in the hope to express themselves in a better sense from digital citizens' point of view, and forever having undeniably producing a 'ripple' effect on moving the boundaries of the media landscape. The cycle of knowledge sharing and representations in this motion therefore are potentially impacting in the shaping of our new knowledge while we human learn how to become 'blended' digital storytellers of the twenty first century.

While the research aims to develop models for digital storytelling in modern world context, it could be equally termed as a challenging preliminary speculation theory to explore on the humanistic aspects of the complex work of digital storytelling for humankind to re-invent new form of "digital universe" (Kaku, 2006, p. 192) and idealistic "civilization" (Whitehead, 1967, pp. 273-274). By examining new ways to bring advancement through the exploration of the arts and scientific knowledge of storytelling expressions and representations processes, humankind are seen as chasing the waves to overcome their critical needs, to re-plot and expand the imaginary digital landscape of now and into the future. This research is challenged to draw critical highlights, exploring new storytelling-knowledge (metaphorical "knowledge-mapping") - a transformational traverse in intellectual search especially in the manifestation of stories creation and development. Content authors and creators are the drivers for key media innovation with potential invention of a new universe powered by human efforts of reengineering process. Our dire aim is to excel in digital storytelling as we transform ourselves in this "manifesting" mode while advancing and expanding our new 'fictionally' bound media channels for audiences of today and tomorrow. Technically, researches perspective will discuss the relevance or how capable we are to 
represent our "new digital universe" knowledge intellectually, whether in objective forms and or with added fictional settings (concepts) and dimensions (interpretation and perceptions) digitally. So in this sense, human will forever be seen as pursuing the game of assuming to balance own capabilities in telling winning-stories in reality. Hence, it is a conceptual anchoring connection for human to realize that they live and drive the process of actualizing the human storytelling experiences in a completely and densely "fluxed" virtual digital landscape.

\begin{tabular}{|c|c|c|}
\hline \multirow{3}{*}{ 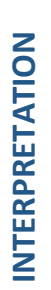 } & \multicolumn{2}{|c|}{$\begin{array}{l}\text { MEDIA KNOWLEDGE IN DIGITAL WORLD: } \\
\text { Where meaning definitions and story engagement process meet }\end{array}$} \\
\hline & \multicolumn{2}{|c|}{ Research objectives and methods list for digital storytelling: } \\
\hline & $\begin{array}{l}\text { 1. Discuss working model for } \\
\text { storytelling problems }\end{array}$ & $\begin{array}{l}\text { To define the meaning of what art in representation of } \\
\text { knowledge is }\end{array}$ \\
\hline \multirow{3}{*}{ 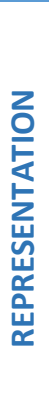 } & $\begin{array}{l}\text { 2. Identifying model for knowledge } \\
\text { gap in storytelling mapping and } \\
\text { processes }\end{array}$ & $\begin{array}{l}\text { To identify methods for appropriating storytelling } \\
\text { expressiveness levels }\end{array}$ \\
\hline & $\begin{array}{l}\text { 3. Developing ideal models of } \\
\text { objectivity vs fictional } \\
\text { experience in storytelling }\end{array}$ & $\begin{array}{l}\text { To perfecting methods for qualifying a "dual-way" } \\
\text { idealization of human storytelling experience }\end{array}$ \\
\hline & $\begin{array}{l}\text { 4. Redefining a new meaning of } \\
\text { the "world" (the digital world) }\end{array}$ & $\begin{array}{l}\text { Models for "idealization" of human fictional } \\
\text { experience across represented medium }\end{array}$ \\
\hline
\end{tabular}

Figure 2 Above listed the research approaches with aligning goals that present research findings in four stages of "media knowledge in digital transformation", targeting to inspire and verify the needs of further study on aspects of positive reinforcement of storytelling experience in various social cultural conditions or situations.

\section{RATIONALE OF STUDY}

\section{Value creations for digital economies and the media needs}

The rationale of the research study are basic theoretical investigations that examine the way of expressions, objective impacts and intellectual work of modern digital storytellers. Drawing on the research knowledge and results from this paper, it is hoped that there will be the needs of development to further discover advanced models as strategic follow up studies in this transformation period. A focused study will be needed. A study that will look at the functional system (media use - audience versus platform of expressions) and entities and regulating bodies (knowledge engagement - authority versus engagement responsibilities) to manage new means of digital content creations and validation claims for industry needs (creative economy) in the domain of practical digital storytelling applications, specifically to drive the future formation and development of a new class of digital civilization.

With the rationale of the current research goal, the following discussion plans will demonstrate a structural theoretical clarifications and case study discussions on interpretations of new concepts and meanings for illustrating and speculating the new imagined media needs to ensure a communication success model could be derived at, with the purpose to support a phenomenally emerging media change in the transformational period of the twenty first century.

\section{DISCUSSIONS}

Three major aspects are identified and discussed based on Figure 1 proposed research approach. Each aspect of discussion attempts to answer and analyze issues with human storytelling experience (reality world) in relations to complexity of media needs (communication needs), to perfect storytelling skills (analysis/working models) in reality world. These aspects are listed in below stages and illustrated in Figure 1: 
Stage 1 (Reality world): Human's interpretation of the world

Stage 2 (Communication needs): Representation and expression of the "self"

Stage 3 (Analysis and working models): Actualizing human experience during stories creation

\section{Stage 1- Discussions on Human-World Rationalization \\ Human's interpretation of the world: Art in representation of knowledge (refer Figure 1 and Figure 2 point 1)}

Why do human invent? And the meaning of creations process to human. As we all know that traditionally art is a form of practice of human expressions. For example, expressions with life and living as creations work such as fashion and costumes, expressions with objects as product design like decorations, expressions towards our environment as beliefs and cultures that we represent knowledge in astonished ambiance of colors reflected on earth. These included tangible entities such as constructed architectural forms, temples, statues of worships and other physical structures. For humankind, all these practices of creations, authorship and expressions are communication forms that bring civilization to human. It contributed profound achievements in human world history. Therefore, it is crucial that we investigate on the intellectual search process of these human creations. From our intellectual involvement as we interpret, describe, transfer knowledge and share, eventually our search actions should benefit from these activities. What is the major concern that is lacking studies in the new media world is the developmental knowledge that looks at the mindset of human activities created and processed by themselves; the authors, creators, and the audience of digital content in our digital world, all works of fictional creations perspectives should be questioned. The mental process of these workmen have set paths and the rediscovery journey of themselves with their creations, on how they reinvent to survive and be successful in telling their own creation stories to audience of the society are the core contextual issues in this research context.

In order to discuss the models for the development of fictional storytelling concepts, the initial phase is to understand the state of our world in relations to how we perceive knowledge and to represent the meaning in making sense of things in our world.

\section{Interpretation and description on mental process representation}

To identify and defining working models, to solve knowledge gap in storytelling and mapping processes for stories representation (refer to Figure 2 point 2), we need to question ourselves on how we see our world, and to investigate the meaning of that interpretation in relations to the world. Therefore, an initial phase of this research study examines specific real world cases. The following work described is to demonstrate and reference the present and past human interpretation and creations examples, looking at how human see the world (perception) and searching meanings (cognition) in relations to interpreting specific social situations in the communication processes. Below are elaborations on some of these related works:

\section{Case description: Storytelling on well-being \\ Sadhguru, the founder of the Isha Foundation, Adiyogi Centre India}

A case description on utilizing power of human imagination, imagining the present (physical existence) and connecting moments (constructing future); from the power of imagining suggestions, to the topic of reengineering human well-being, Sadhguru, as the world renowned activist in human well-being not only has created a tremendously impactful work of "storytelling" act of art practice. He played a critical worldly role in shaping the mindset of his society from where he belonged. Millions support were also drawn from global audience visited his country to attend his inspirational and transformational teachings in inner-engineering programs.

Referring to the mysterious outlook of the statue or sculpture found in Figure 3, where Sadhguru stood (picture right). The statue created by him represents a prominent landmark of significance of "anchoring connection" (TedxSaltLakeCity, 2016) to the Isha Foundation followers. We know that a sign for which human has created, is to cast motivation to our audience imagination. The significance of sign has both an indication of certainty or probability in its primary sense; its form of its relation with what it represents is then the relating elements in its relational studies for elements such as, "conveniency, emulations, sympathy and similitude" as researched by 
Foucault (2002, pp. 65-66). The speculation thinking comes into picture when we realize from this case study that design decisions of the statue, as an "anchored connection" motif to its followers, will then need to accommodate its own power of significance to our reality world. Its significance, effecting on relational elements mentioned here as the acronymic termed "C-E-S-S" (simplified from Foucault's term), will impact on human perception of the world. Therefore, the meaning of human intuition-expressions in live telecast recorded examples from ritual MahaShivRatri festival celebration is in fact, an evidential case of human history in utilizing technology to process digital storytelling and to manifest modes of communicating (expressing) themselves 'fictionally' in represented fictional (pictorial) world across via actual stories manipulation act. The Aiyogi leader strategically demonstrated an imagined future with his original intention to connect with the world intuitively (oral expression) and physically-expressively (dancing act) in his followers' presence during the event activities captured in live telecast delivered over social media.

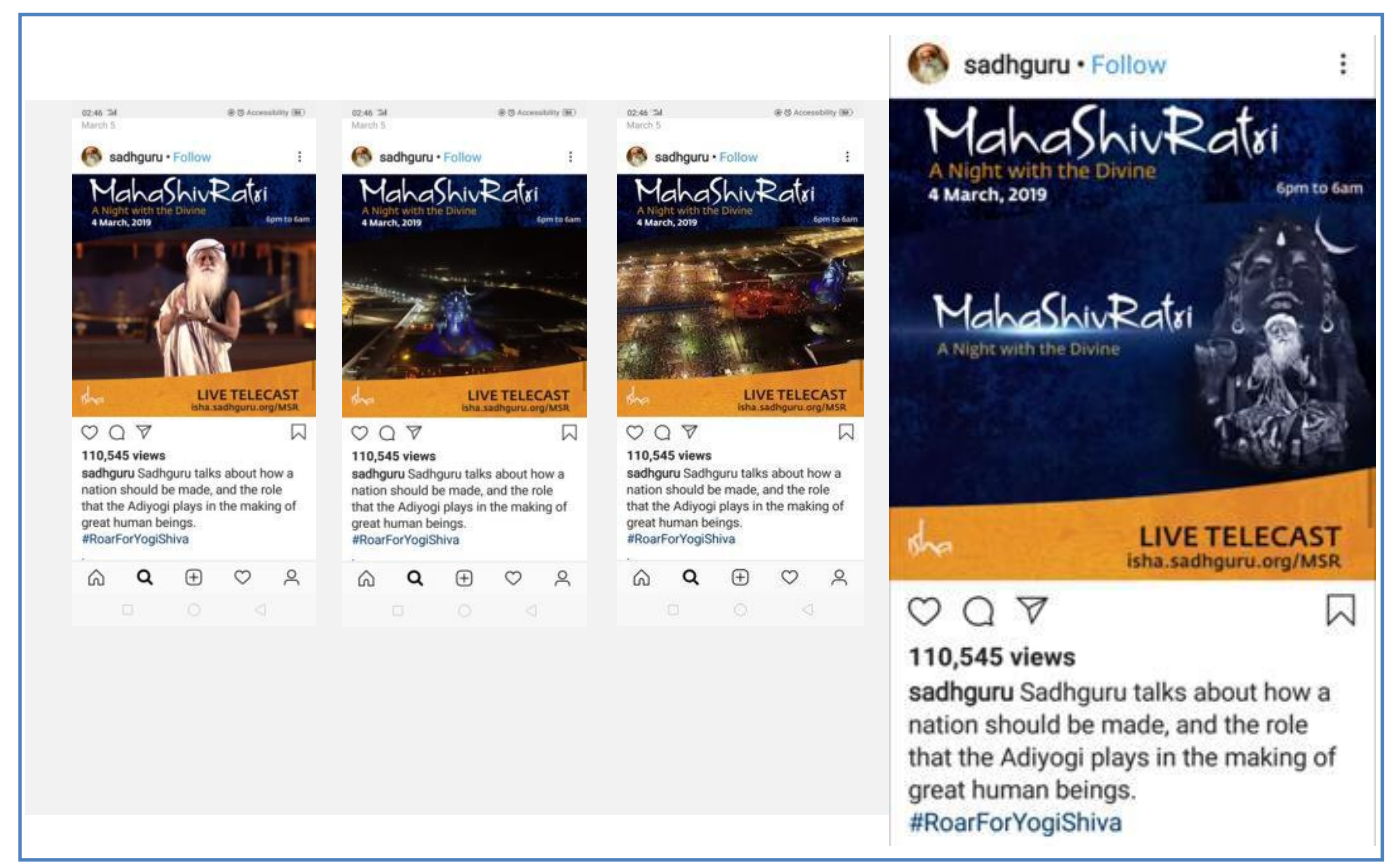

Figure 3 Art in representation of knowledge: A case example to demonstrate the connection of the practice of rituals as repeated actions in expressing human intention, connecting self with both physical and inner worlds, thereby demonstrating the success of digital storyteller's effort in associating "mind-body-space-energy" as a way of "anchoring on connection".

Images above are sources from Sadhguru's Instagram timeline. He is world renowned yogi and the founder of The Isha Foundation at Adiyogi Centre India (an institute of well-being). As a storyteller of human well-being, audience has connected with the presence of his storytelling performance and dances of ritual acts during the celebration night for MahaShivRatri on 4 March 2019, themed “A Night with the Divine” (Sadhguru, 2019).

\section{Stage 2 - Discussion on Stories-Audience Engagement Process}

Representation and the expression of "self" in the world: Embedding storytelling expressiveness levels (refer Figure 1 and Figure 2 point 2)

The act of human imagination is by actual fact an act of connecting our mind to our existence in this physical world. It is an intersection of mindfulness practice in visualizing ourselves in relations to virtual existence in our own "re-imagined world". The stories representation that is reinvented in our mind in this case, is the preconceived impression prior to our expressions of intuition to interact and manipulate the medium for our storytelling engagement process. In order to define knowledge in storytelling process, we have to question and 
search for the meaning of truth in reality of our physical existence. This should bring us a step closer in forwarding our imagination to represent the imprint of that mental process in the world where we live in via medium manipulation. We do not just simply create; we should question the dignity of our conceptual mapping of meaning in order to tap into and capture inspirations from our mental imprint through the act of storytelling via the medium. Reasoning and logical design thinking should be applied on how the crafting of the stories content to be manipulated. So we firstly have to agree within ourselves to express them as materialized objective outcome for our audience view.

"The objective approach to concepts leads to the view that, beyond the perceptual level, knowledge is the grasp of an object through an active, reality-based process chosen by the subject." (Peikoff, 1993, p. 116)

Also, realistically and fictionally speaking, it is concerned with how much tones, shades or lights, density, textures and layers we need to put in our design efforts for that masterpiece of our imagination. It will definitely not be an accidental creation but a carefully mapped process and work that resulted from our intellectual search while we materialize the relevant mental stories representation across our medium. The lesson we should learn from storytelling representation and mapping processes is therefore self-explanatory in Lewis's quote as he stated that "Reason is the natural organ of truth; but imagination is the organ of meaning" (philosophybits, n.d.).

The mapping work therefore suggests that creators should elaborate and examine mindfulness in human expressions and manifestation of the representation of "self" against our environment or objects while we are in stories making and mapping processes. Modern contextual study involves some content engagement and design thinking that encourage audience to connect with our stories content. Audience engagement therefore will make the flux of (creators) our imagination even more seamless and profound in reinforcing stories expression. The following case will describe, examine and clarify further the needs of inspirations within our design thinking of rediscover new challenges of "acting on expressions" in stories content structure for storytelling and representation.

\section{The “Acting on Expressions" Challenges}

"The content of expression itself derives from the impressions which the mind gathers from practical life ie., from its impulses, its desires, and its sensory awareness [...] because of feeling, an image can become an intuition. With this achievement art becomes a symbol of feeling". (Croce, in Hofstadter, et. al. 1964, pp. 555-556)

\section{Case Description: Storytelling for creative industry}

The next intellectual inquiry is on understanding the power of imagination and "adventuring ideas" to create and bring richness for stories content and it is what I referred it as "acting on expressions" challenges. So the next questions is, can we imagining tomorrow (stories from our mind) and at the same time be as real as our reimagined future (actualizing storytelling experience)? Here I propose the next question that will lead us to wonder how objectivity in fictional balance would be achieved while authors and stories creators model and structure their work, attempting and acting on the expressions challenges (see Table 1, the "C-E-S-S" working model is proposed to speculate on the creators' input and perspective in this sense).

\section{Kendy Mitot, an indigenous artist and researcher: Ritual-painting storytelling}

Images below (Figure 4) demonstrated a pictorial sequence of ritual-painting storytelling process. Artist immersed himself in his own imagination, working on perfecting the art of ritual-painting and drawing on intuitiveness when crafting the masterpiece. The core of storytelling task is exercising on artist's conscious effort in maintaining expressiveness level while processing his surrealistic painting strokes on the canvas. 


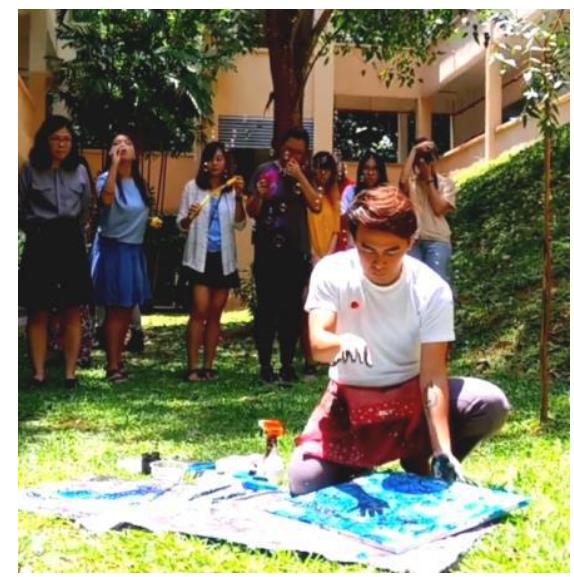

(4a)

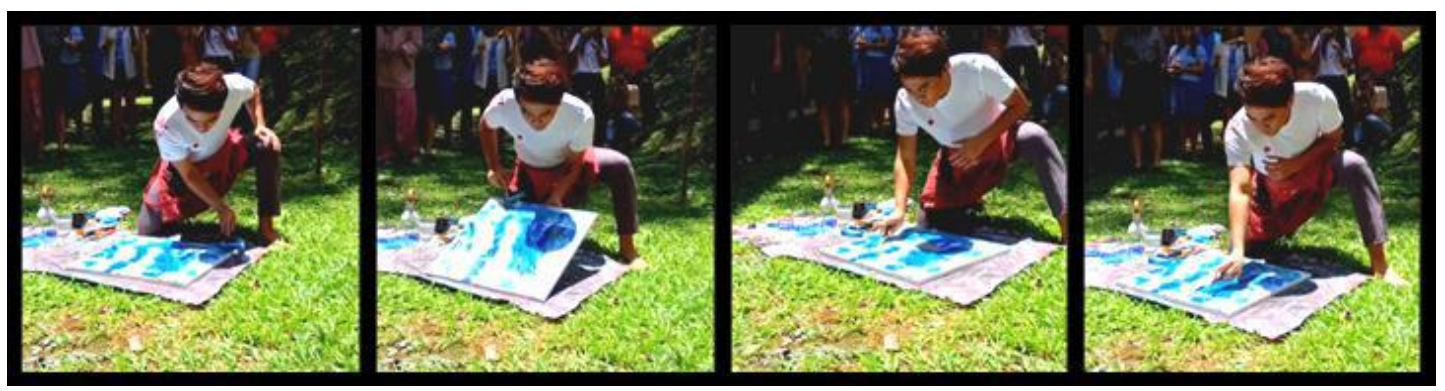

$(4 b)$

Figure 4(a), 4(b): A video (or image) shows a live demo-painting performed by Kendy Mitot, an indigenous artist: An example of ritual painting-storytelling with expressiveness intention actualizing on storytelling experience. In creative industry, methods of performing rituals with painting are rarely used in the sense that it depends on how well the story engagement production could be, to demonstrate the depth in emulations experience as a form of stories expressions level (see Table 1 [y1]).

Stage 3 - Discussion on Working Models and Analysis Study on Real-World, Fictional Stories Representation Model analysis for fictional storytelling: Perfecting methods and idealization of representation in storytelling experience across medium (refer Figure 1 and Figure 2 point 3 and 4)

Table 1 : "Meaning of the world" model: A proposed working model (the "C-E-S-S") for the study of "the order of things" in the creation of our future digital world; focused on creators' input and or the designers' decision to determine its stories appropriateness and expressiveness levels impacting audience over the media platform.

\section{STORYTELLING ENGAGEMENT MAPPING AND \\ ITS RELATIONAL ELEMENTS FOR STORYTELLING PROCESS \\ A working Model for Creators' Input in Storytelling:}

\begin{tabular}{|l|l|l|}
\hline Convenience (C) & Framing situations & $(\mathbf{x 1 )}$ \\
\hline Emulations (E) & Constructing expressiveness levels & $\mathbf{( y 1 )}$ \\
\hline Sympathy (S) & Stories intention vs. meaning validation & $\mathbf{( z 1 )}$ \\
\hline Similitude (S) & Creations of "higher-self", the new digital universe & $\mathbf{( s t 1 )}$ \\
\hline
\end{tabular}




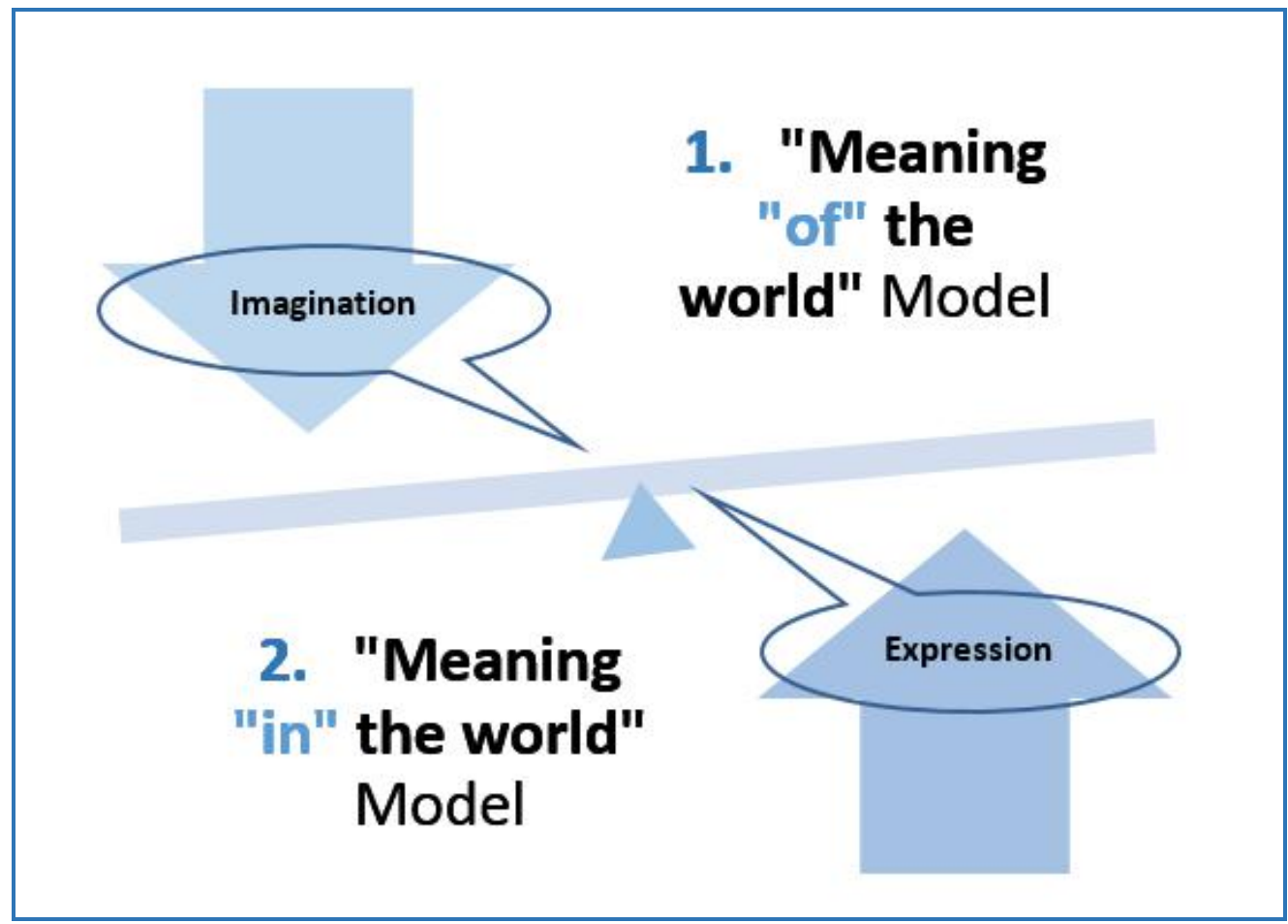

Figure 5 "Acting on Expressions" Challenges: A model illustrated presents the mirrored form of conceptual and imaginary lever-indicator, illustrating situations at the instance when we perceive (interpret) the reality world at point [1] in contrast to fictional world as "represented" stories meaning at point [2] delivered as content across platform.

A supporting claim or statement can be derived based on the model's concept reflected meaning. For example, during the instance when storytellers express and draw on the audience sympathy (see Table 1 (z1)), they are recognizing the needs of searching for an "anchor of connection" (TedxSaltLakeCity, 2016) by reinforcing situational intention while acting on meaning of human actualizing their experience for expressions. This relationship takes place when any incidental happenings between the relations of human in relating "self" in the world while they are seeking to define meaning in their world during the actualizing of storytelling representation and expressions challenges process.

From expression challenges to "actualization" of storytelling experience with expressive intention (refer Figure 1 and Table 2 y2 and $z 2$ )

To work of digital storytellers is an involvement of themselves as creators to manifest the state identified in Table 1 [st1] that represents the state of storytelling with similitude to bring forward an experience of creating a higher "self" that resembles the intentional creation of a new digital universe in reality world or the current state that they are in. To achieve the higher state, a critical conditioning of digital landscape will then be initiated with ourselves as creators, recognizing the changing-needs (refer Figure 1) in today's digital media landscape so that we will then be able to take advantage of the media "of-the-now" while expressing and delivering media (stories) for tomorrow's audience. Below is an intermediary model realized and proposed to clarify the needs to crystallize our mental process with intention to promote that seamless expression of 'higher-self creations' when we actualize digital storytelling development experience across our represented medium. 


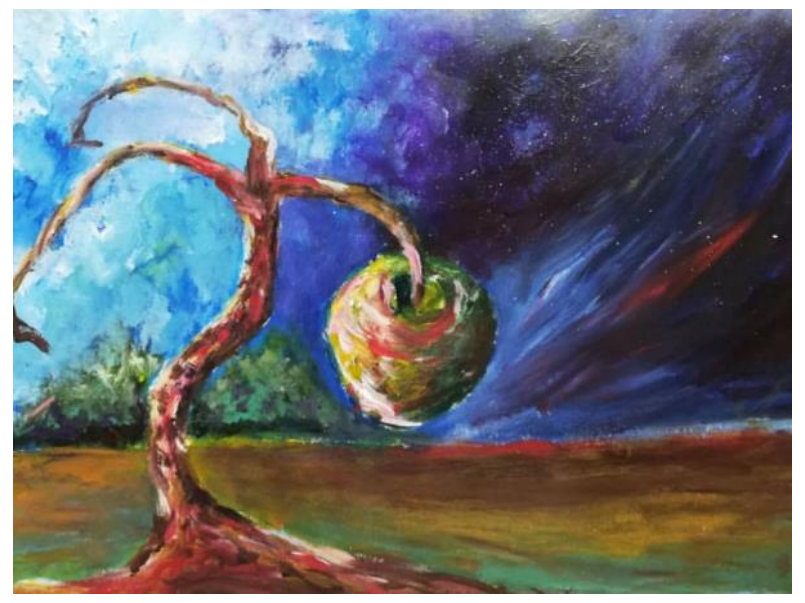

Figure 6 As human search for the knowledge in seeing, and in mirroring self in that "attached" stories: Image source titled "Tree of Roots" (Expressions: Nature, Roots and Ritual World, 2019), is a work of art painting by Mitot to express surrealistic impressionism with scenic nature featuring symbol of roots depicting the mythical ritual world. Its magical appeal of earthly imagination formed the basis of constructed working models that aim to define new meaning in stories conceptualization processes for digital storytellers. Metaphorical meaning of the image interpretation is modelled and clarified in Table 2 as below.

Table 2: "Meaning of the world" model: The proposed working model of the "C-E-S-S" for the study of "the order of things" for the creation of our future digital world that will focus on creator's input and designers' decision to determine its stories appropriateness and expressiveness levels for audience interpretation over digital media platform.

\begin{tabular}{|l|l|l|}
\hline \multicolumn{2}{|c|}{ ACTUALIZING HUMAN STORYTELLING EXPERIENCE IN } \\
STORIES REPRESENTATION PROCESS \\
A working Model for Storytellers Acting on Expressions Challenges: \\
\hline 2'Apple' from the past & Framing situations & $(\mathbf{x})$ \\
\hline 'Apple' in the present & Constructing expressiveness levels & $(\mathbf{y 2})$ \\
\hline 'Apple' in the future & Stories intention vs. meaning validation & $\mathbf{( z 2 )}$ \\
\hline 'Apple' for the future perfect & Creations of "higher-self", the new digital universe & $\mathbf{( s t 2 )}$ \\
\hline
\end{tabular}

\section{Future concept of digital universe (refer Table 2 [st2], Figure 7 number 4)}

In Table 2, the "C-E-S-S" concept is a developing model for me to work on the speculation and clarification of its deeper meaning in terms of its futuristic digital world context. In the work of Kaku (2006, pp. 230-231), he mentioned about the "Holographic Universe" and described his concept about the features and navigation of and within that holographic universe. He explained that not only our digital future should look like two dimensional digital landscape of today with layers or "shadow" (p. 231), it will also resemble the existing form of computer programs that we have. Can this new concept of our new digital universe be an inspiration for digital storytellers to imagine a futuristic picture that will represent our future "designer universe" (pp. 241242), and yet be filled with human anxiety to try to understand and discover what makes the core study of "holographic" communication principles interesting in near future?

\footnotetext{
${ }^{2}$ Refer Table 2, the "Apple" in this context is a metaphorical image for imagination in the digital world landscape scenarios where mobile technology could support the integration of a seamless digital creations experience. What inspirations could be we draw on the future of digital storytelling process in our imagined near future?
} 


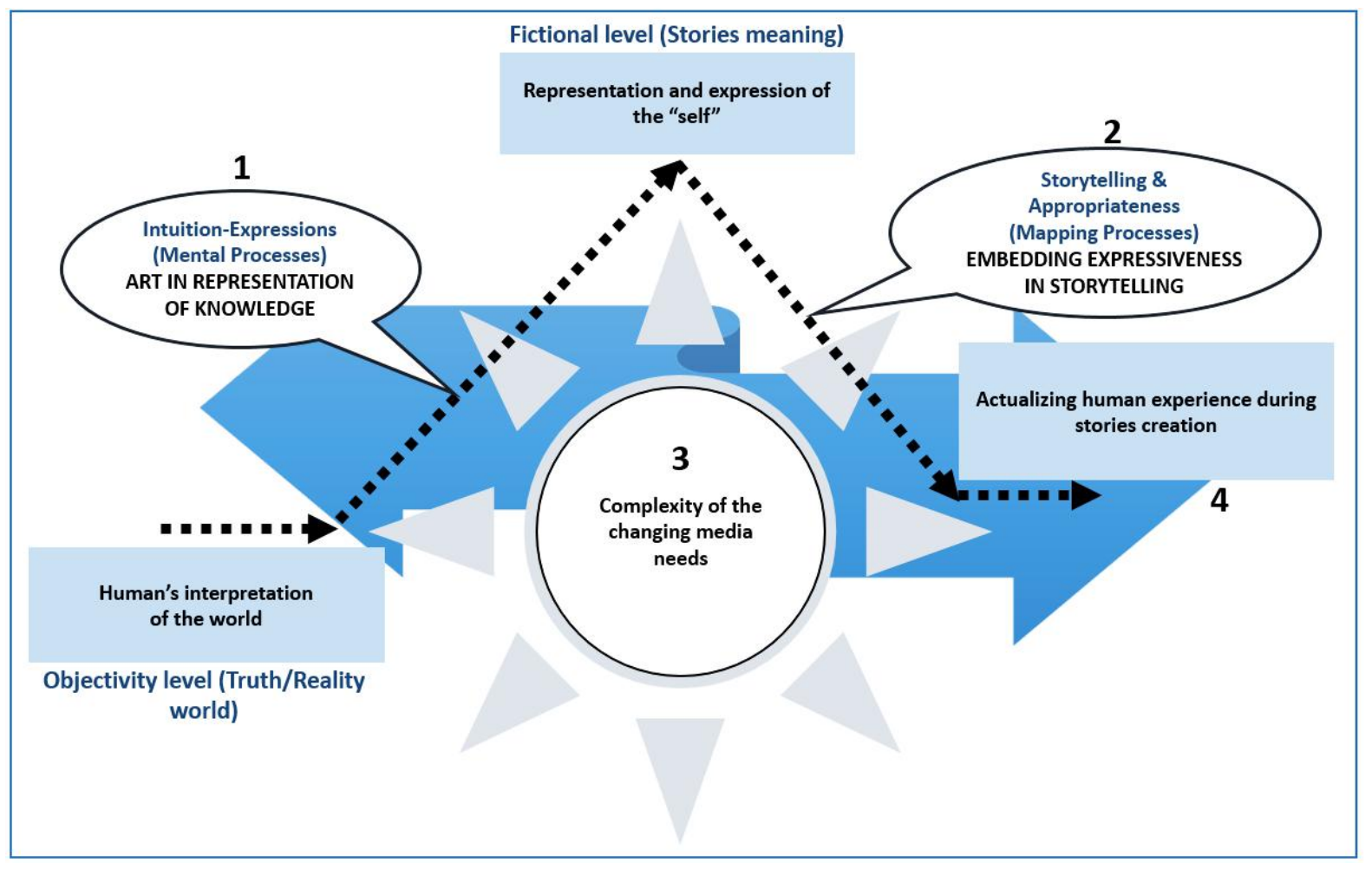

Figure 7 Adventures of "ideas" in storytelling processes - This figure shows and suggests the need for critical conditioning of digital landscape (as indicated in the stated phase numbered [3]), where humankind as digital audience taking the role of inventors in leading change in media needs for shared-stories creations in the state of digital transformation and digital "universe" manifestation process.

\section{Actualizing human experience during stories creation}

The last section of this paper is to address the meaning of actualization process of storytelling. It is a design thinking process about choice of relevant content approach to express stories content. Therefore, logically we are engaging in mapping the level of objective and fictional experiences at both sides of creation process. This will allow creators or storytellers judging on approach of expression, combined with a sense of mental conceptualizing on the ways to establishing and qualifying a "dual-way" idealization of human storytelling experience (refer Figure 8, value creations mental model). 


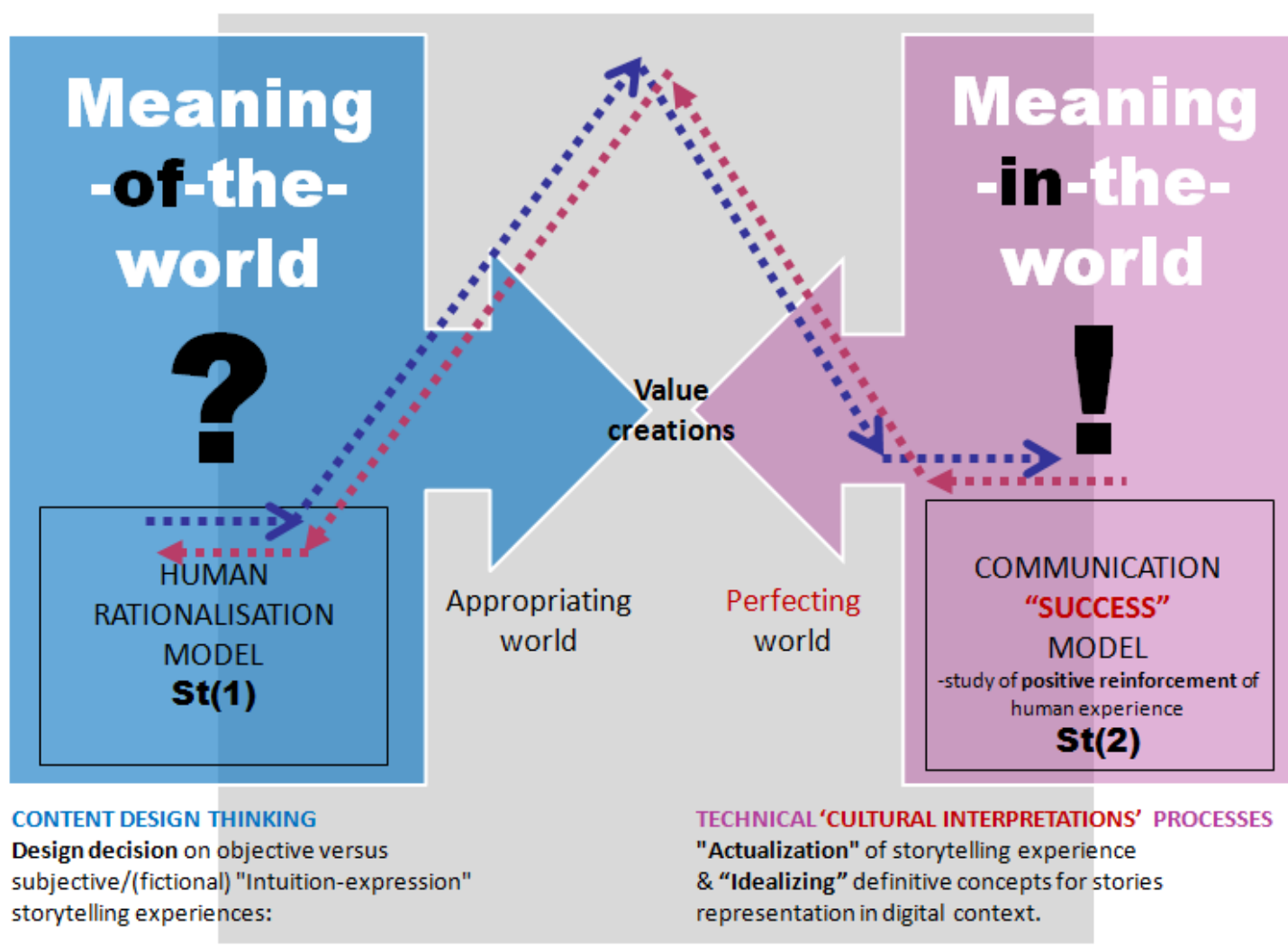

Figure 8 Value creation for digital economies: The figure shows a dual way qualifying mental processes of rationalization and representations for storytellers. To perfect their stories content the issues discussed included in this paper Section 2 and Section 3 are crucial for development, in terms of "perfecting" our digital world, hence bring values to our creations and added values to digital economies from audience consumption and marketing development perspectives.

Any Rand Objectivism theory (Peikoff, 1993, p. 117) mentioned that "objective" is the state of mind for which if one is specifically refers to the manipulation of a conceptual process as during the actualization of human storytelling experience, it is the conscious effort of our mind interplay with human consciousness. It signifies certain level of human survival needs as expressions of stories based on "objectivity". Objectivity then means careful selection of some sort of edited thoughts that should tell a metaphorical consistency with that of reality, thus mapping process accuracy is rationalized in near perfect level in human stories creation and representation in digital world context.

\section{SUMMARY OF FINDINGS}

In summary, all discussions and analysis on the concepts and meanings of storytelling in relations to three categorized stages of discussions are presented as various theoretical and philosophical objectivity study in making sense of fictional digital storytelling experience to audience in the reality world. The findings are summarized in the following perspectives:

Four major stages presented in overall illustrations are visualized, contained and re-illustrated as in Figure 8, to bring highlights on the concept of our conscious effort and proposing the needs to work on: (1) adventuring our pre-conceived ideas about our world, and (2) to search for methods in our expressions, thereby crafting and rediscover, and (3) develop better pathway to close the gap between storytellers (creators' input) mental model and, considering (4) the prevailing digital economy climate exerting manifestation and effects on media audience (audience interpretation on content) who are at the perception end, interacting with our embedded 
stories content across medium. Overall, these four steps processes of communication success model are briefly summarized as below:

1) ADVENTURES - Desires to control world.

Human intention in representing knowledge and appropriating storytelling, searching for meanings in reality world;

\section{2) STORYTELLING METHODS - Giving meaning to world.}

From seeing the world to searching for knowledge in seeing and interpreting world, mapping processes are definitive to stories representation;

3) PATHWAY SOLUTIONS - The variables manifestation.

The media change is in fact evolutionary due to the concept of open innovation in current digital economy climate (Kaku, 2017). It will have impact on technical cultural interpretation processes of the media elements. This assumption draws on the complexity of changing media needs of today, within the digital landscape (as indicated in the left/right horizontal expanding arrows of Figure 7); Some effect will also be on the rising (1. creation) actions and falling (2. precision/appropriation) actions as indicated in the improvised Freytag's Pyramid model (see Figure 1) which suggested that humans are forever assuming their capabilities in balancing the "dual-way" storytelling forces;

4) NEW ECONOMY CLIMATE - Values creations in digital economies.

Moving from the anxiety of content design concepts of appropriating our world (Figure 8), via actualizing the storytelling experience process, it is assumed that all content stories meanings (indicated as the central top point illustrated in Figure 8) will draw us closer in developing the shift to maximize humankind's power, acting on advancing digital storytelling profession as a form of language (new "storytelling" language), driving modern content makers in constant intentional tasks of gauging what makes the best means of expressions and representation in new generation economic context.

One has to recognize new possibilities in communication methods and facility tools as our media advantages of today that we will have. Our current and future needs should focus on "perfecting" (referred by Whitehead as "The Eros" (1967, p. 275) and realizing our storytelling rituals as an evergreen craft-in-the-making to "renew" our storytelling experiences. By doing this, we are indirectly impacting and shaping our digital futures. In view of the needs to rediscover new digital content strategies and achieving better rewards in value creations processes, our digital world outlook in reality world should be directing us yet to perform further levels of intellectual search on screen-based cultural practices.

\section{CONCLUSION}

As a conclusion, this research of theoretical and contextual study has presented and clarified that a new class of information communication model will be needed. A model that may act as potential indicator to discuss and provide further study on potential impact of current major changing needs; that is the needs in recognizing media not only as "windows" for stories expressions to audience. While the highlights of this phase of research studies aim at giving constructive input on subjective issues that designers and marketing content developers are facing in current context of expanding digital landscape, it is suggested that value creations are indeed a series of conscious effort and work to direct attention given by global social content creators. Together, the elements of media, content, and audience will always be the right pathway of charting new information input as all future shared content developed for our global audience. Therefore, the new re-imagined future of our new digital universe should inspire our further work, looking forward to re-position digital content "design thinking" as "sub-elements" for media change. We will need all new strategies that could possibly drive creative digital economies to a higher dimension to compensate new values creation as digital economic growth in near future. 


\section{Future Work}

Much future work will be initiated in near time speculating on the impact of new content design thinking resulting from various stages of structured stories meanings. It should be exciting to look at the strategies for humankind to create new concepts for platform sharing and audience interaction perspectives with the content. Thus, a cycle of new knowledge generation effecting on future digital "civilization" (Whitehead, 1967, pp. 191, 273-274) will lead to a new direction for the next stage of research.

\section{ACKNOWLEDGEMENT}

The author would like to express gratitude to TIIKM in making it possible to create a new positive outlook for an Asian research culture. Author felt fortunate to be able to participate in MEDCOM 2019 and supported by a well-managed and prestigious institute. Author would also like to thank Mitot, an indigenous artist of Sarawak Bidayuh culture for his contribution in sharing his inspirational painting work mentioned in this paper. It is hoped that more young generation will take up the challenging role of modern researchers to drive a common goal with the urge to study and redefine new meanings of future "digital civilization" in this historical digital transformation era.

\section{References}

Croce, B. (1976). Shaftesbury: Selections from "Aesthetics" (Encyclopaedia Britannica, Fourteenth Edition). In R. K. Albert Hofstadter, \& R. K. Albert Hofstadter (Ed.), Philosophies of Art and Beauty: Selected Readings in Aesthetics from Plato to Heidegger (Paperback edition ed., pp. 555-556, 559-560, 561-562, 566). Chicago, United States of America: The University of Chicago Press.

Foucault, M. (2002). The Order of Things: An archaeology of the human sciences (First edition ed.). London, United Kingdom: Routeledge Classics.

Harari, Y. N. (2017). Homo Deus: A Brief History of Tomorrow (First Edition ed.). Vintage, London, UK: Penguin Random House.

Kaku, M. (2017, November 10). Michio Kaku - Where Will The Digital Economy Take Us? (C. Kaku, Producer) Retrieved April 28, 2019, from Youtube: https://youtu.be/K1EZWYqm-5E

Kaku, M. (2006). Parallel Worlds: The Science of Alternative universes and our future in the Cosmos. London: Penguin Books.

Mitot, K. Tree of Roots. Tunku Abdul Rahman University College, Kuala Lumpur, Malaysia.

Peikoff, L. (1993). Objectivism: The Philosophy of Ayn Rand (Vols. VI, The Ayn Rand Library). New York, United States of America: Meridian.

Philosophybits. (n.d.). Philosophy Bits. Retrieved October 28, 2018, from Philosophy Bits: https://www.philosophybits.com/tagged/C.-S.-Lewis

Sadhguru. (2019, March 4). MahaShivRatri 2019, 4th Mar 2019: A night with the Divine. Retrieved March 30, 2019, from Isha: https://isha.sadhguru.org/mahashivratri/

TedxSaltLakeCity. (2016, October 7). Ted.com, Tedx. (Tedx, Editor, \& TedxSaltLakeCity) Retrieved May 6, 2019, from TEDx Talks: http://youtu.be/KSXh1YfNyVA

Whitehead, A. N. (1967). Adventures of Ideas. In A. N. Whitehead, Adventures of Ideas (First Free Press Paperback Edition ed., pp. 191, 241-243, 273-274, 275). London, New York, United States of America: Macmillan Company. 